ORIGINAL RESEARCH

\author{
C. Garel \\ I. Cont \\ C. Alberti \\ E. Josserand \\ M.L. Moutard \\ H. Ducou le Pointe
}

\title{
Biometry of the Corpus Callosum in Children: MR Imaging Reference Data
}

\author{
BACKGROUND AND PURPOSE: The availability of data relating to the biometry of the CC in children that \\ are easy to use in daily practice is limited. We present a reference biometry of the CC in MR imaging \\ in a large cohort of children.
}

MATERIALS AND METHODS: Cerebral MR imaging studies of children with normal examination findings were selected retrospectively. Children born preterm and those with or at risk of developing cerebral malformations were excluded. The following parameters were measured: FOD, APD, LCC, GT, BT, IT, $\mathrm{ST}$, and the S/T. Inter- and intraobserver agreement and sex effect were evaluated.

\begin{abstract}
RESULTS: Six hundred twenty-two children were included (320 boys, 302 girls), ranging from 1 day to 15 years of age. Normal values (from the 3rd to 97th percentile) are provided for each parameter. All parameters showed rapid growth up to 3 years of age followed by slower (FOD, APD, LCC, GT and ST) or absent (S/T) growth. Growth of BT and IT was completed by 7-8 years. CC modeling (IT/ST) was completed by 3 years. FOD was larger in boys from the age of 1 year (statistically significant). The other parameters did not show any sex effect. Inter- and intraobserver agreement was excellent for all parameters except for IT.
\end{abstract}

CONCLUSIONS: As measured, our data result in easy and reproducible MR imaging biometry of the CC in children.

ABBREVIATIONS: APD = anteroposterior diameter of the corpus callosum; BT = body thickness; $\mathrm{CC}=$ corpus callosum; $\mathrm{Cl}=$ confidence interval; $\mathrm{EN}=$ exponential normal; $\mathrm{FOD}=$ fronto-occipital diameter; GT = genu thickness; ICC = intra-class correlation coefficient; IT = isthmus thickness; LOA = limits of agreement; LCC = length of the corpus callosum; ST = splenium thickness; $\mathrm{S} / \mathrm{T}=$ position of the splenium related to the tegmentum

I:

$n$ most cases, the morphologic approach is sufficient to ensure the normality of the $\mathrm{CC}$ on the midsagittal section acquired with MR imaging. However, in certain cases, due to physiologic variations in CC morphology, analysis of the CC may be more difficult; these variations cause problems, especially in the setting of neuropsychiatric disorders, dysmyelinating and demyelinated disorders, pre- and perinatal trauma, or hypoxic injury. Moreover, the CC undergoes morphologic changes throughout infancy, which must be taken into account for proper evaluation. Review of the pediatric literature does not provide biometric standard reference data of the CC that are easy to use in daily practice.

The aims of this study were to provide reference biometric data of the CC in MR imaging from a large cohort of children, to evaluate the position of the splenium in relation to the brain stem, to describe morphologic changes of the CC throughout childhood and particularly throughout infancy, to evaluate a possible sex effect on CC biometry and morphology, and, finally, to evaluate the reproducibility of CC biometry in MR imaging.

\section{Received October 19, 2010; accepted after revision December 11.}

From the Service de Radiologie (C.G., I.C., H.D.L.P.) and Service de Neuropédiatrie (M.L.M.), AP-HP, Hôpital d'Enfants Armand-Trousseau, Paris, France; Unité d'Epidémiologie Clinique (C.A., E.J.), AP-HP, Hôpital Robert Debré, Paris, France; Université Paris Diderot (C.A.), Paris VII, France; Institut National de la Santé et de la Recherche Médicale (C.A.), CIC-EC, CIE 5, Paris, France; and Université Pierre et Marie Curie (H.D.L.P.), Paris VI, France.

Please address correspondence to Catherine Garel, MD, Department of Radiology, Hôpital d'Enfants Armand-Trousseau, 26 Avenue du Dr Arnold Netter, Paris, France; e-mail: catherine.garel@trs.aphp.fr

Indicates article with supplemental on-line tables.

http://dx.doi.org/10.3174/ajnr.A2542
In the future, a good knowledge of the evolution of CC biometry throughout childhood will make it possible to correlate morphologic and biometric abnormalities of the CC with the clinical status of the patient.

\section{Materials and Methods}

Cerebral MR imaging examinations were selected retrospectively in the MR imaging data base of our hospital from 2005 to 2008. Approval of the institutional review board was obtained; informed consent from the parents was not required.

The examinations were included in the study when a midline sagittal section acquired with a $\mathrm{T} 1$ or $\mathrm{T} 2$ sequence was available, in the absence of motion artifacts that rendered the measurements inaccurate, and in the absence of cerebral abnormalities and premature birth.

Children between 1 day and 15 years of age were included until the final number of subjects was reached. They were randomly selected in increments of 2 months to 1 year of age so that distribution of age groups could be about the same.

The MR imaging examinations were performed in children presenting with findings possibly associated with cerebral pathology: headache, seizures, myoclonia, dizziness, balance disorders, abnormal visual findings, deafness, small size, precocious puberty, facial palsy, velar insufficiency, and nasal or scalp midline mass or cyst without any cerebral abnormality.

Children with metabolic disorders, neuropsychological disorders, cerebral malformation, pre- and perinatal trauma or hypoxic injury, and multiple extracerebral malformations and those born preterm were excluded from the study even if no cerebral abnormalities were detected. 
Table 1: Different biometric parameters analyzed in the study ${ }^{\mathrm{a}, \mathrm{b}}$

\begin{tabular}{|c|c|c|c|}
\hline Abbreviation & Parameter & Definition & Fig \\
\hline APD & Anteroposterior diameter of the CC & Distance between the anterior aspect of the genu and the posterior aspect of the splenium & $1 \mathrm{~A}$ \\
\hline LCC & True length of the CC & Curvilinear distance between the rostrum and the splenium at midthickness of the CC & $1 B$ \\
\hline GT & Thickness of the genu of the CC & Thickness of the CC, measured at the level of the genu & $1 C$ \\
\hline BT & Thickness of the body of the CC & Thickness of the CC, measured at the level of the body & $1 C$ \\
\hline IT & Thickness of the isthmus of the $\mathrm{CC}^{\mathrm{a}}$ & Thickness of the CC, measured at the level of the isthmus & $1 C$ \\
\hline ST & Thickness of the splenium of the CC & Thickness of the CC, measured at the level of the splenium & $1 C$ \\
\hline FOD & Fronto-occipital diameter ${ }^{\mathrm{b}}$ & Distance between the extreme points of the frontal and occipital lobes & $1 E$ \\
\hline$S / T$ & Distance splenium/tegmentum & $\begin{array}{l}\text { Distance at the level of the fastigium, between a line drawn along the dorsal surface of } \\
\text { the brain stem and another line parallel to the first one and passing through the level of } \\
\text { the most posterior point of the splenium }\end{array}$ & $1 F$ \\
\hline
\end{tabular}

${ }^{a}$ When the isthmus could not be identified because of insufficient CC modeling, the IT was measured at the level where the fornix abuts the CC (CC-fornix junction) (Fig 1D).

${ }^{b}$ Sometimes the frontal and occipital lobes were not visible on the midsagittal section due to enlargement of the interhemispheric fissure. The FOD was then acquired on a slightly parasagittal section.
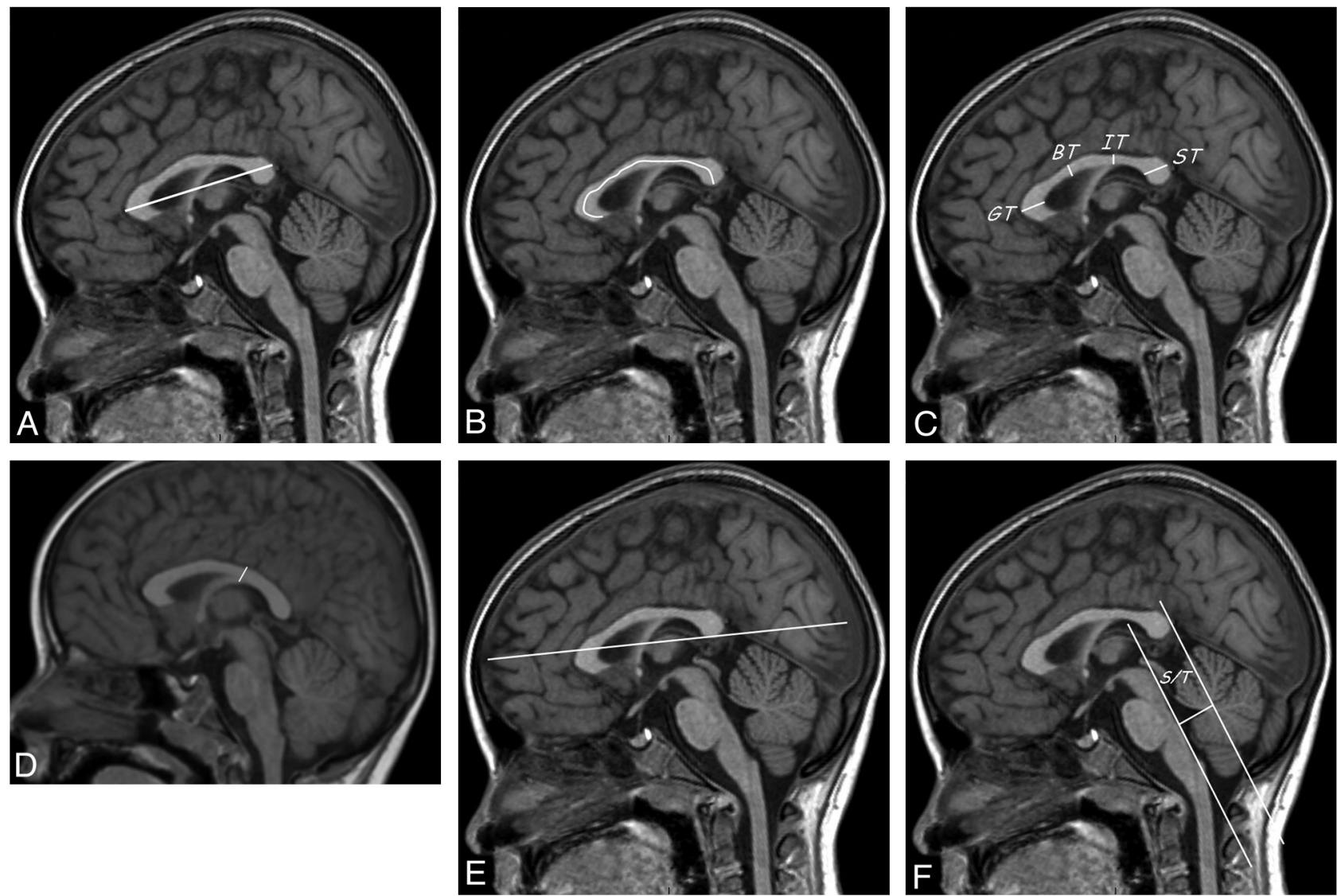

Fig 1. Description of the different biometric parameters measured with MR imaging. $A$, Measurement of the APD of the CC, the distance between the anterior aspect of the genu and the posterior aspect of the splenium. $B$, Measurement of the true LCC, the curvilinear distance between the rostrum and the splenium at midthickness of the CC. $C$, Measurement of the thickness of the CC, at the level of the genu (GT), body (BT), isthmus (IT), and splenium (ST). D, measurement of the IT when the isthmus could not be identified because of insufficient CC modeling. IT was measured at the level where the fornix abuts the CC (CC-fornix junction). E, Measurement of the FOD, the distance between the extreme points of the frontal and occipital lobes. $F$, Evaluation of the position of the splenium. A line was drawn along the dorsal surface of the brain stem. Another line was drawn parallel to the first one and passing at the level of the most posterior point of the splenium. The $S / T$ distance between those lines was measured at the level of the fastigium.

\section{Procedure}

MR imaging was performed on a 1.5T unit (Achieva; Philips Healthcare, Best, the Netherlands). Midline sagittal sections were obtained with T1- (section thickness $=1.5-4 \mathrm{~mm}$ ) or T2-weighted (section thickness $=2.5-4 \mathrm{~mm}$ ) sequences.

All biometric data were acquired on digital images. The different biometric parameters analyzed are summarized in Table 1 and illustrated in Fig 1.

The interobserver reproducibility of the measurements was evaluated by 2 radiologists (I.C., C.G.) on a sample of a total number of 50 children, chosen at random within each age group. Each radiologist was unaware of the results obtained by the other one. Moreover, the intraobserver reproducibility was evaluated in the same sample by 1 of the 2 radiologists (C.G.).

\section{Statistical Analysis}

Qualitative variables are described as frequency (percentage). Estimates of ICCs and Bland-Altman plots were used to explore intraand interagreement between the 2 radiologists. Agreement was considered slight when the ICC was $\leq 0.2$, fair when $0.2<$ ICC $\leq 0.4$, moderate when $0.4<\mathrm{ICC} \leq 0.6$, substantial when $0.6<\mathrm{ICC} \leq 0.8$, and almost perfect (excellent) with the ICC $>0.8$. 
Age-specific reference intervals for biometric parameters were estimated by using the parametric method described by Royston and Wright. ${ }^{1}$ Briefly, a centile curve is estimated by using the formula:

$$
C_{p}=\mu_{T}+q_{p} \sigma_{T},
$$

where $q_{p}$ is the corresponding centile of the standard Gaussian distribution, $T$ is continuous age expressed in years, and $\mu_{T}$ and $\sigma_{T}$ indicate the corresponding values of mean and SD for each curve at age $T$.

Initially, a running line smoother of the measurement of interest $Y$ against age $T$ depending on sex provides an impression of the shape of the mean curve and also examines the need to model each curve separately by sex (On-line Tables 1-11). It shows that measurements of interest tend to an asymptote as age increases. It is then desirable that the functions for the curves flatten. Such behavior is induced by applying the following exponential transformation to age:

$$
X=\exp \left(\frac{T-T_{1}}{T_{n}-T_{1}} \log (\rho)\right),
$$

where $T_{1}$ and $T_{n}$ denote minimum and maximum ages, respectively, and $\rho$, a preselected constant equal to 0.01 . $X$ is further used in place of $T$ in regression models for the parameter curves.

Initial Box-Cox or natural logarithmic transformation is applied if required to reduce positive skewness and heteroscedasticity of the measurement of interest $Y$. A fractional polynomial of degree $m$ is then fitted by least-squares regression of the measurement of interest against age to create a suitable function for $\mu_{X}$, with the powers as numbers chosen from the set $\{-2,-1,-0.5,0,0.5,1,2,3\}$, where $X^{0}$ denotes a natural logarithm of $X$. An appropriate fractional polynomial is selected by comparing the difference in deviance between models having degrees $m$ and $m+1$ by using a $\chi^{2}$ variate on $2 d f$. Sometimes, several models may fit approximately equally well, in which case the final choice is based on nonstatistical grounds. The fitted values from the regression give the estimated mean curve and the "scaled absolute residuals," $\sigma_{X}$. If the scaled absolute residuals appear to show no trend with age, the SD is estimated as that of the residual of the measurement of interest from the regression on age; otherwise fractional polynomial regression analysis is performed to estimate an appropriate curve in the same way as for the mean.

The model fit is assessed by calculating the SD scores ( $z$ score) as

$$
z=\frac{Y-\mu_{X}}{\sigma_{X}} .
$$

The ordered $z$ scores are plotted to provide a graphic check of normality. If normality is accepted, no further modeling is required. If after this step, the normality is not reached, an exponential transformation of Manly statistics is applied resulting in an age-related skewness curve for $z$ also fitted by maximum likelihood. This conducts to an EN model and the estimation of the centile by

$$
C_{p E N}=\mu_{X}+\mu_{X} \frac{\log \left(1+\gamma_{X} q_{p}\right)}{\gamma_{x}},
$$

with $\gamma_{X}$ being inversely related to the skewness. As $\gamma_{X} \rightarrow 0$, the EN distribution tends to normal.

Goodness-of-fit methods used were Q tests exploring the moments of the $z$ scores and permutation bands, which are a graphic way

\begin{tabular}{|c|c|c|c|}
\hline $\begin{array}{l}\text { Age Class } \\
\text { in Years }\end{array}$ & $\begin{array}{c}\text { Males } \\
(n=320)\end{array}$ & $\begin{array}{c}\text { Females } \\
(n=302)\end{array}$ & $\begin{array}{c}\text { All } \\
(N=622)\end{array}$ \\
\hline$<1$ & 65 & 51 & 116 \\
\hline $1-2$ & 26 & 32 & 58 \\
\hline $2-3$ & 32 & 27 & 59 \\
\hline $3-4$ & 39 & 23 & 62 \\
\hline $4-5$ & 15 & 12 & 27 \\
\hline $5-6$ & 15 & 13 & 28 \\
\hline $6-7$ & 15 & 14 & 29 \\
\hline $7-8$ & 15 & 16 & 31 \\
\hline $8-9$ & 10 & 22 & 32 \\
\hline 9-10 & 9 & 19 & 28 \\
\hline 10-11 & 12 & 18 & 30 \\
\hline 11-12 & 18 & 14 & 32 \\
\hline 12-13 & 14 & 15 & 29 \\
\hline 13-14 & 16 & 13 & 29 \\
\hline 14-15 & 19 & 13 & 32 \\
\hline
\end{tabular}
of examining the adequacy of the models. ${ }^{2}$ When a model EN is chosen, the graphic check of normality of residuals is performed on

$$
U=\frac{\exp \left(\gamma_{X} z\right)-1}{\gamma_{x}}
$$

Note that $Y$, the measurement of interest in the $z$ score calculation, corresponds to the transformed value if a preliminary one has been done.

Estimated centiles and reference intervals are calculated by substituting the fitted curves of the mean and SD into the equation of $C p$ or $C P E N$, according to whether skewness has been modeled. When the variable being modeled $Y$ is initially Box-Cox transformed, centile curves on the original scale are obtained by applying a back-transformation to the calculated curves,

$$
C_{\text {original scale }}=\left[1+\theta C_{p}(X)\right]^{\frac{1}{\theta}}
$$

with $\theta$ being the parameter of the Box-Cox transformation.

Analyses were computed on SAS, Version 9.1 (SAS Institute, Cary, North Carolina) and STATA, Version 9.0 (StataCorp, College Station, Texas) software.

\section{Results}

\section{General Characteristics}

We included 622 children, from 1 day to 15 years of age. The distribution of the number of children as a function of age and sex is described in Table 2. There were 320 boys and 302 girls.

\section{Biometry and Sex Effect}

Normal values (from the 3 rd to 97 th percentiles) are provided for each parameter in On-line Tables 1-11 and are depicted in Fig 2. Moreover, normal values (3rd, median, and 97 th percentiles) are summarized in Table 3 . All parameters showed rapid growth until 3 years of age; then, their growth slowed down (FOD, APD, LCC, GT, ST) or stopped (S/T). Growth of BT and IT was completed by 7-8 years of age.

Loess smoothing curves of the different parameters, depending on age (in years) and sex, are provided in Fig 3 (Online Tables 1-11).

Sex effect was only present for FOD, with the measurement being higher in boys compared with girls. 


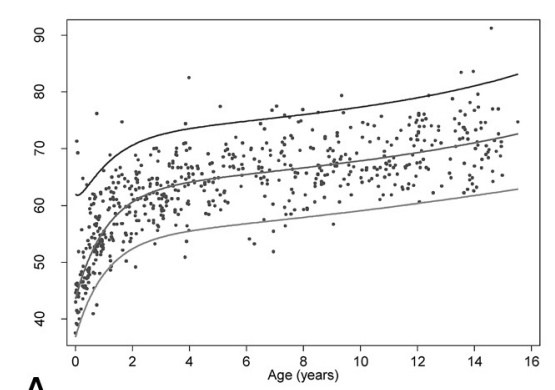

A

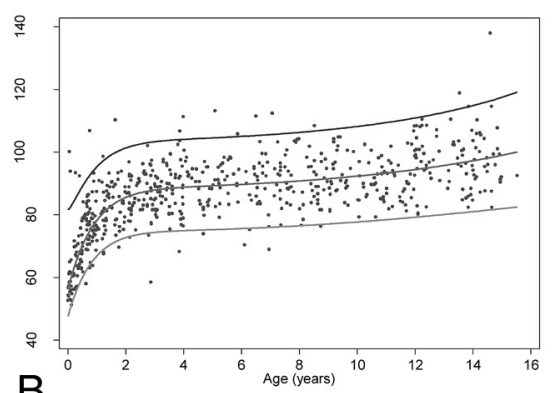

B

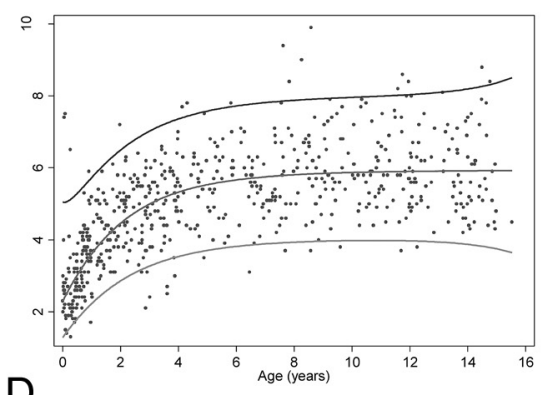

D

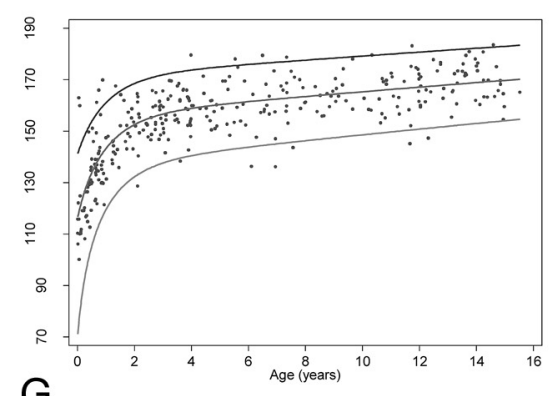

G

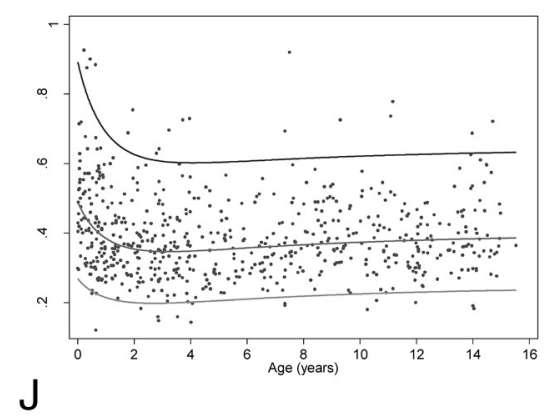

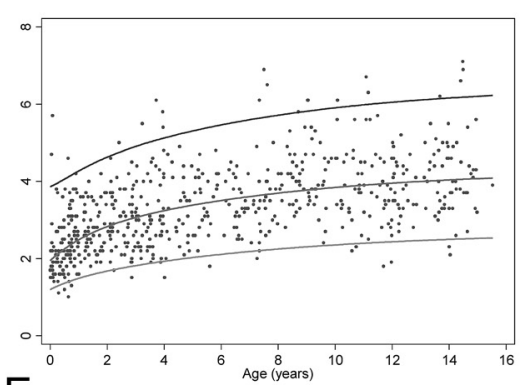

E

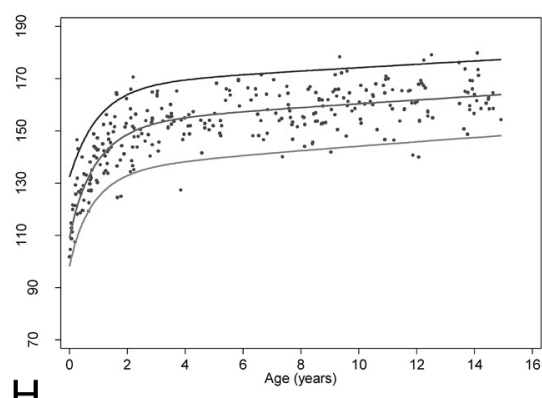

$\mathrm{H}$

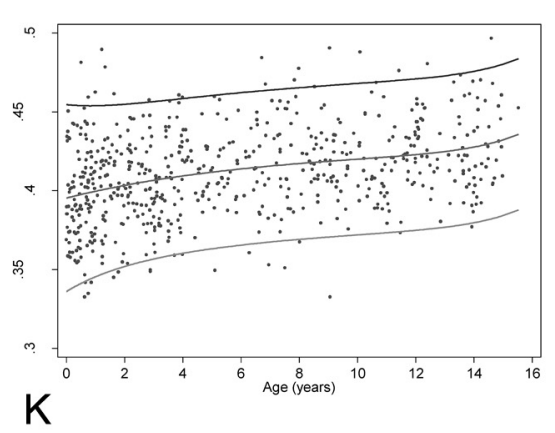

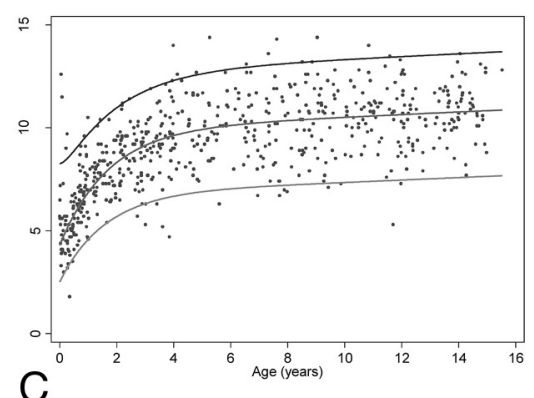

C
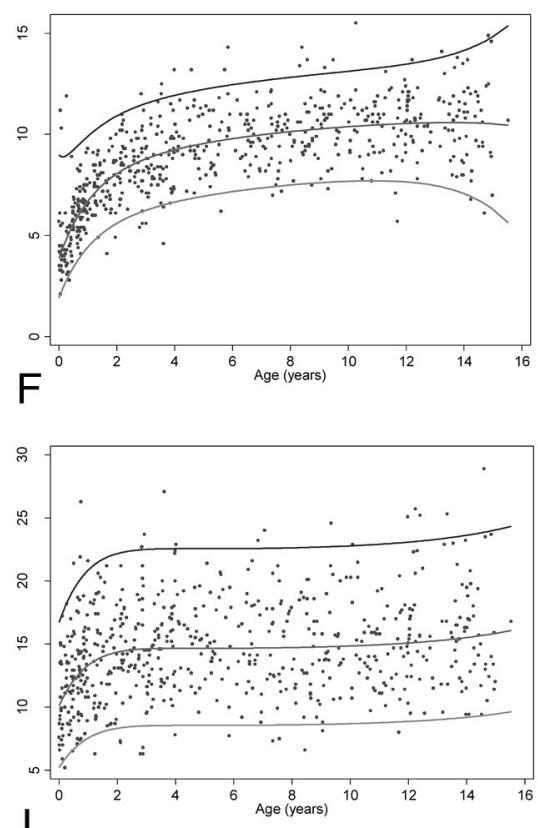

I

Fig 2. Reference intervals (3rd, 50th, 97th) for the different parameters: $A=A P D ; B=L C C ; C=G T ; D=B T ; E=I T ; F=S T ; G=F O D$ for males; $H=F O D$ for females; $I=S / T$; $\mathrm{J}=\mathrm{IT} / \mathrm{ST}$; and $\mathrm{K}=\mathrm{ADP} / \mathrm{FOD}$.

\section{Reproducibility}

Intra- and interobserver agreement was almost perfect for all parameters except for IT (Table 4), with a correct 95\% LOA for the mean bias estimates.

\section{Models}

Eleven models were built: Six measurements needed prior Box-Cox transformation, and 7 models were EN, whereas 4 other models were normal. The equations for estimating reference values for the different parameters are presented in Table 5 (On-line Tables 1-11).

\section{Morphology}

The IT/ST ratio progressively decreased until 3 years of age and then became stable. This result indicates that CC modeling was completed by 3 years of age.

\section{Discussion}

This study provides current reference values on the distribution of the parameters of the CC in children from birth to 15 years. The statistical method used was one of those recommended by the World Health Organization Multicenter Growth Reference Study Group. ${ }^{3}$ We used the method of Royston and Wright ${ }^{2}$ pro- 


\begin{tabular}{|c|c|c|c|c|c|c|c|c|c|c|c|c|c|c|c|c|c|c|c|}
\hline \multirow[b]{2}{*}{ Percentile } & \multicolumn{19}{|c|}{ Age (yr) } \\
\hline & 0 & 0.5 & 1 & 1.5 & 2 & 2.5 & 3 & 4 & 5 & 6 & 7 & 8 & 9 & 10 & 11 & 12 & 13 & 14 & 15 \\
\hline \multicolumn{20}{|l|}{ APD } \\
\hline $3 r d$ & 36.8 & 43.7 & 47.9 & 50.6 & 52.4 & 53.6 & 54.4 & 55.5 & 56.2 & 56.8 & 57.4 & 57.9 & 58.5 & 59.1 & 59.7 & 60.4 & 61 & 61.7 & 62.5 \\
\hline Median & 43.6 & 50.9 & 55.6 & 58.6 & 60.6 & 61.9 & 62.9 & 64.1 & 64.8 & 65.5 & 66 & 66.6 & 67.2 & 67.9 & 68.6 & 69.3 & 70.1 & 71 & 72 \\
\hline 97th & 62 & 63.9 & 66.8 & 69.1 & 70.6 & 71.7 & 72.5 & 73.5 & 74.2 & 74.8 & 75.4 & 76 & 76.6 & 77.3 & 78.1 & 79 & 79.9 & 81.1 & 82.3 \\
\hline \multicolumn{20}{|l|}{ LCC } \\
\hline 3rd & 47.6 & 60 & 66.9 & 70.7 & 72.7 & 73.8 & 74.4 & 75 & 75.3 & 75.6 & 76 & 76.5 & 77.1 & 77.7 & 78.4 & 79.2 & 80.1 & 81 & 82 \\
\hline Median & 56.3 & 70.2 & 78.3 & 82.9 & 85.5 & 87 & 87.9 & 88.7 & 89.2 & 89.7 & 90.2 & 90.8 & 91.5 & 92.3 & 93.3 & 94.4 & 95.7 & 97.2 & 99 \\
\hline 97th & 81.7 & 89.1 & 95.3 & 99.1 & 101.3 & 102.6 & 103.3 & 104.1 & 104.5 & 105 & 105.6 & 106.3 & 107.1 & 108.2 & 109.4 & 110.9 & 112.7 & 114.8 & 117.5 \\
\hline \multicolumn{20}{|c|}{ 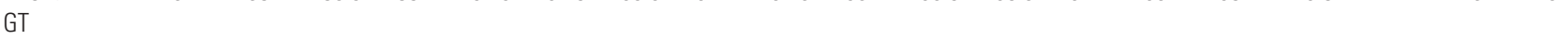 } \\
\hline 3rd & 2.5 & 3.7 & 4.6 & 5.2 & 5.7 & 6 & 6.3 & 6.7 & 6.9 & 7 & 7.1 & 7.2 & 7.3 & 7.3 & 7.4 & 7.5 & 7.5 & 7.6 & 7.6 \\
\hline Median & 4.3 & 5.8 & 6.9 & 7.7 & 8.3 & 8.8 & 9.1 & 9.6 & 9.9 & 10.1 & 10.2 & 10.3 & 10.4 & 10.5 & 10.6 & 10.6 & 10.7 & 10.8 & 10.8 \\
\hline 97th & 8.3 & 8.9 & 9.7 & 10.4 & 11 & 11.4 & 11.8 & 12.3 & 12.6 & 12.9 & 13 & 13.1 & 13.2 & 13.3 & 13.4 & 13.5 & 13.5 & 13.6 & 13.7 \\
\hline \multicolumn{20}{|l|}{ BT } \\
\hline 3rd & 1.3 & 1.8 & 2.2 & 2.6 & 2.9 & 3.1 & 3.3 & 3.5 & 3.7 & 3.8 & 3.9 & 3.9 & 4 & 4 & 4 & 4 & 3.9 & 3.9 & 3.7 \\
\hline Median & 2.3 & 3 & 3.6 & 4.1 & 4.5 & 4.8 & 5 & 5.3 & 5.5 & 5.7 & 5.8 & 5.8 & 5.8 & 5.9 & 5.9 & 5.9 & 5.9 & 5.9 & 5.9 \\
\hline 97th & 5 & 5.3 & 5.7 & 6.1 & 6.5 & 6.8 & 7 & 7.4 & 7.6 & 7.7 & 7.8 & 7.9 & 7.9 & 8 & 8 & 8 & 8.1 & 8.2 & 8.4 \\
\hline \\
\hline $3 r d$ & 1.2 & 1.4 & 1.5 & 1.6 & 1.7 & 1.7 & 1.8 & 1.9 & 2 & 2.1 & 2.2 & 2.2 & 2.3 & 2.4 & 2.4 & 2.4 & 2.5 & 2.5 & 2.5 \\
\hline Median & 1.9 & 2.2 & 2.5 & 2.7 & 2.8 & 3 & 3.1 & 3.2 & 3.4 & 3.5 & 3.6 & 3.7 & 3.8 & 3.8 & 3.9 & 4 & 4 & 4 & 4.1 \\
\hline 97th & 3.9 & 4.1 & 4.3 & 4.5 & 4.6 & 4.8 & 4.9 & 5.1 & 5.3 & 5.5 & 5.6 & 5.7 & 5.8 & 5.9 & 6 & 6 & 6.1 & 6.2 & 6.2 \\
\hline \multicolumn{20}{|l|}{ ST } \\
\hline 3rd & 1.9 & 3.4 & 4.4 & 5.1 & 5.6 & 6 & 6.2 & 6.7 & 6.9 & 7.2 & 7.4 & 7.5 & 7.6 & 7.7 & 7.7 & 7.7 & 7.5 & 7.1 & 6.3 \\
\hline Median & 3.9 & 5.6 & 6.7 & 7.5 & 8.1 & 8.5 & 8.8 & 9.2 & 9.5 & 9.8 & 10 & 10.1 & 10.3 & 10.4 & 10.5 & 10.5 & 10.6 & 10.6 & 10.5 \\
\hline 97th & 9 & 9.2 & 9.9 & 10.5 & 10.9 & 11.3 & 11.5 & 11.9 & 12.2 & 12.5 & 12.7 & 12.8 & 13 & 13.1 & 13.3 & 13.5 & 13.7 & 14.1 & 14.8 \\
\hline \multicolumn{20}{|l|}{ FOD boys } \\
\hline 3rd & 69.8 & 105.2 & 119.5 & 127.4 & 132.3 & 135.4 & 137.6 & 140.5 & 142.3 & 143.8 & 145.1 & 146.3 & 147.5 & 148.6 & 149.7 & 150.8 & 151.9 & 153 & 154 \\
\hline Median & 116.3 & 133.9 & 143.3 & 148.9 & 152.5 & 154.9 & 156.6 & 158.8 & 160.3 & 161.5 & 162.5 & 163.4 & 164.4 & 165.3 & 166.2 & 167.1 & 168 & 168.9 & 169.7 \\
\hline 97th & 141 & 153.7 & 161.1 & 165.6 & 168.5 & 170.5 & 171.9 & 173.7 & 175 & 175.9 & 176.8 & 177.6 & 178.4 & 179.2 & 180 & 180.7 & 181.5 & 182.2 & 183 \\
\hline \multicolumn{20}{|c|}{ (2) } \\
\hline $3 r d$ & 98.1 & 115.9 & 124.7 & 129.8 & 132.9 & 134.9 & 136.3 & 138.2 & 139.5 & 140.6 & 141.5 & 142.4 & 143.3 & 144.2 & 145 & 145.8 & 146.7 & 147.5 & 148.3 \\
\hline Median & 109 & 128.5 & 138.7 & 144.8 & 148.7 & 151.2 & 152.9 & 155 & 156.3 & 157.3 & 158.1 & 158.9 & 159.6 & 160.4 & 161.1 & 161.8 & 162.5 & 163.2 & 163.9 \\
\hline 97th & 132.5 & 146.5 & 155.2 & 160.5 & 163.8 & 166.1 & 167.6 & 169.5 & 170.6 & 171.5 & 172.2 & 172.9 & 173.5 & 174.2 & 174.8 & 175.5 & 176.1 & 176.8 & 177.4 \\
\hline \multicolumn{20}{|c|}{ 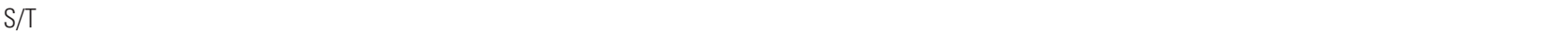 } \\
\hline $3 r d$ & 14 & 22.9 & 26.6 & 28.6 & 29.8 & 30.4 & 30.9 & 31.4 & 31.8 & 32.2 & 32.4 & 32.6 & 32.8 & 32.9 & 33 & 33 & 33 & 33 & 33.1 \\
\hline Median & 22.6 & 28.9 & 32 & 33.8 & 34.8 & 35.4 & 35.8 & 36.4 & 36.8 & 37.1 & 37.4 & 37.6 & 37.8 & 37.9 & 38 & 38.1 & 38.1 & 38.1 & 38.1 \\
\hline 97th & 34.7 & 37.3 & 39 & 40 & 40.7 & 41.1 & 41.4 & 41.8 & 42.2 & 42.5 & 42.8 & 43.1 & 43.3 & 43.4 & 43.5 & 43.6 & 43.7 & 43.7 & 43.7 \\
\hline \multicolumn{20}{|l|}{ Ratio IT/ST } \\
\hline 3rd & 0.27 & 0.23 & 0.21 & 0.2 & 0.2 & 0.2 & 0.2 & 0.2 & 0.21 & 0.21 & 0.21 & 0.22 & 0.22 & 0.22 & 0.23 & 0.23 & 0.23 & 0.23 & 0.23 \\
\hline Median & 0.49 & 0.42 & 0.38 & 0.36 & 0.35 & 0.35 & 0.35 & 0.35 & 0.35 & 0.36 & 0.36 & 0.37 & 0.37 & 0.37 & 0.38 & 0.38 & 0.38 & 0.38 & 0.38 \\
\hline 97th & 0.89 & 0.76 & 0.69 & 0.65 & 0.63 & 0.61 & 0.61 & 0.6 & 0.6 & 0.61 & 0.61 & 0.61 & 0.62 & 0.62 & 0.62 & 0.63 & 0.63 & 0.63 & 0.63 \\
\hline \multicolumn{20}{|c|}{ Ratio APD/FOD } \\
\hline 3rd & 0.34 & 0.34 & 0.35 & 0.35 & 0.35 & 0.35 & 0.36 & 0.36 & 0.36 & 0.37 & 0.37 & 0.37 & 0.37 & 0.37 & 0.37 & 0.37 & 0.38 & 0.38 & 0.38 \\
\hline Median & 0.4 & 0.4 & 0.4 & 0.4 & 0.4 & 0.41 & 0.41 & 0.41 & 0.41 & 0.41 & 0.42 & 0.42 & 0.42 & 0.42 & 0.42 & 0.42 & 0.42 & 0.43 & 0.43 \\
\hline 97th & 0.45 & 0.45 & 0.45 & 0.45 & 0.45 & 0.46 & 0.46 & 0.46 & 0.46 & 0.46 & 0.46 & 0.47 & 0.47 & 0.47 & 0.47 & 0.47 & 0.47 & 0.48 & 0.48 \\
\hline
\end{tabular}

posing a completely parametric method, namely the fractional polynomials and exponential transformation.

The CC is the main commissure connecting the 2 cerebral hemispheres. It is a late-maturing structure. Its development has been studied in detail by Rakic and Yakovlev. ${ }^{4}$ Although controversial, it may be summarized as follows: at 6- to 8 -weeks' postconceptual age, the more dorsal part of the lamina reuniens (the superior part of a small amount of tissue bridging the midline) thickens. Part of it will become the CC. At 11- to 12-weeks' postconceptual age, interhemispheric crossing fibers are observed in the region of the genu. The development of the CC progresses in a craniocaudal direction. The rostrum develops last at 18 - to 20 -weeks' postconceptual age. ${ }^{5}$ Evaluation of the anteroposterior diameter of the CC with fetal MR imaging from 26 weeks' gestation shows a progressive growth of the CC. ${ }^{6}$
In the $\mathrm{CC}$, the fibers undergo a posterior-to-anterior maturation, and in neonates, the fractional anisotropy is higher in the splenium compared with the genu. ${ }^{7}$ During the first 24 months, the fractional anisotropy rapidly increases in the genu and the splenium. It shows relative stability in the splenium after 2 years of age, while in the genu, it increases more slowly until 3 years of age. Similarly, the apparent diffusion coefficients show rapid and then slower decrease in these areas. ${ }^{8}$

The callosal fibers connect primary sensory-motor or associative areas. Their size and degree of myelination depend on their location. The large fibers are mostly located in the isthmus (motor, somatosensory, and auditory cortex) and in the posterior splenium (visual cortex). The thin fibers are mostly located in the genu and in the anterior splenium (prefrontal and temporoparietal associative areas). ${ }^{9}$

Postnatal changes are largely determined by the degree of 

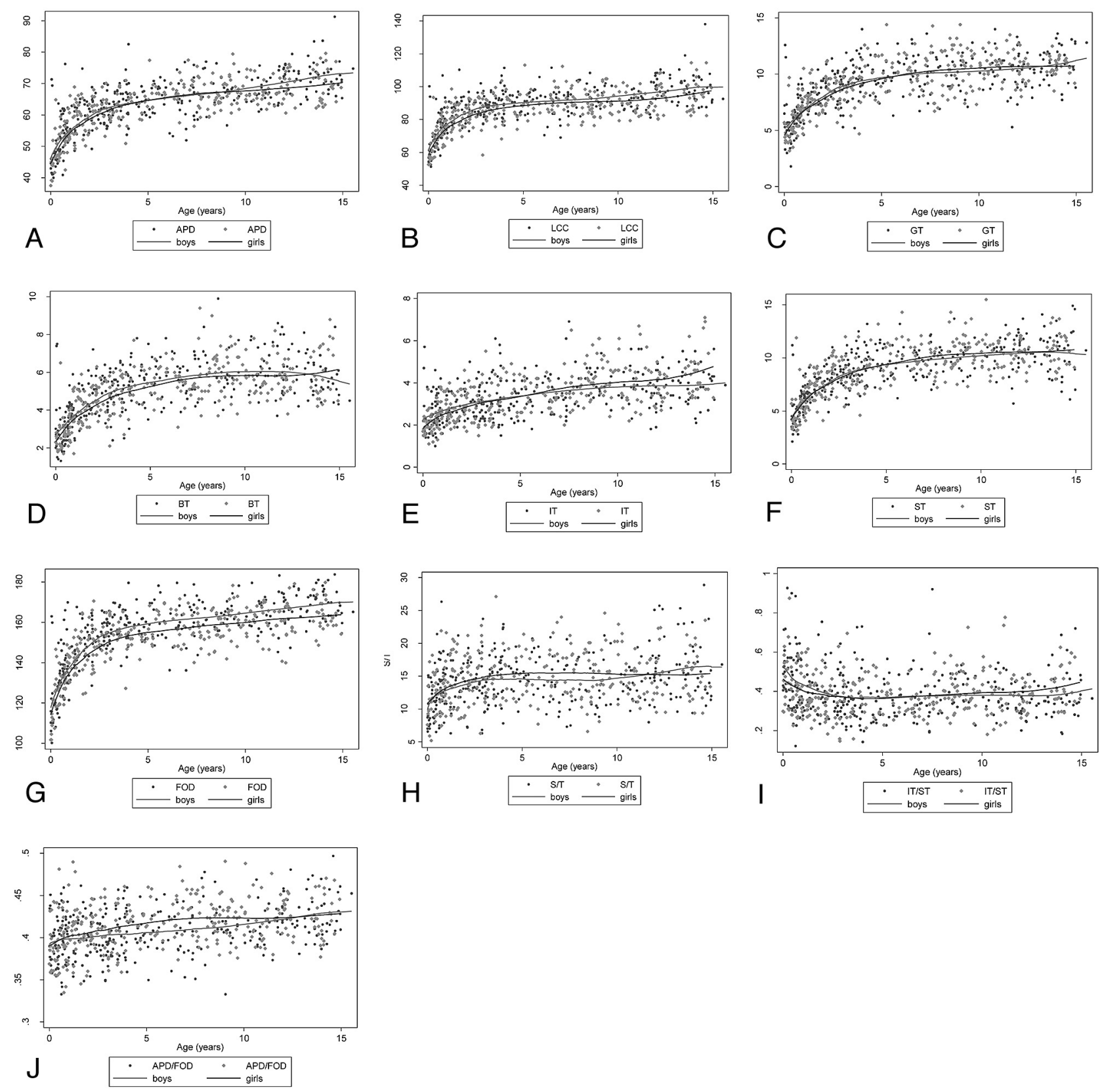

Fig 3. Loess smoothing curves of the different parameters depending on age (years) and sex. $A=A P D ; B=L C C ; C=G T ; D=B T ; E=I T ; F=S T ; G=F O D ; H=S / T ; I=I T / S T$; $J=A D P / F O D$

\section{Table 4: Agreement of cerebral biometric MR imaging parameters}

\begin{tabular}{|c|c|c|c|c|c|c|}
\hline \multicolumn{4}{|c|}{ Interobserver Agreement } & \multicolumn{3}{|c|}{ Intraobserver Agreement } \\
\hline $\begin{array}{l}\text { Agreement/ } \\
\text { Parameter }\end{array}$ & Mean Bias & $95 \%$ LOA & ICC $(95 \%$ CI) & Mean Bias & $95 \%$ LOA & ICC $(95 \% \mathrm{CI})$ \\
\hline APD & -0.08 & $(-1.70-1.53)$ & $0.995(0.991-0.997)$ & 0.12 & $(-1.11 ; 1.35)$ & $0.997(0.995-0.998)$ \\
\hline LCC & 4.40 & $(-1.62-10.32)$ & $0.88(0.80-0)$. & -0.22 & $(-4.49 ; 4.06)$ & $0.98(0.96-0.99)$ \\
\hline GT & 0.07 & $(-1.46-1.61)$ & $0.95(0.91-0.97)$ & 0.42 & $(-0.98-1.82)$ & $0.94(0.90-0.97)$ \\
\hline BT & 0.02 & $(-1.67-1.71)$ & $0.83(0.73-0.90)$ & 0.03 & $(-1.20-1.27)$ & $0.91(0.85-0.95)$ \\
\hline IT & 0.14 & $(-1.44-1.72)$ & $0.72(0.56-0.83)$ & -0.02 & $(-1.09-1.06)$ & $0.87(0.78-0.92)$ \\
\hline ST & -0.15 & $(-1.16-1.99)$ & $0.92(0.87-0.96)$ & 0.36 & $(-2.57-3.28)$ & $0.80(0.67-0.88)$ \\
\hline FOD & 2.90 & $(-6.23-12.11)$ & $0.93(0.89-0.96)$ & 0.64 & $(-6.64-7.92)$ & $0.97(0.95-0.98)$ \\
\hline$S / T$ & -0.03 & $(-3.06-3.00)$ & $0.89(0.82-0.94)$ & 0.16 & $(-1.98-2.30)$ & $0.93(0.88-0.96)$ \\
\hline
\end{tabular}




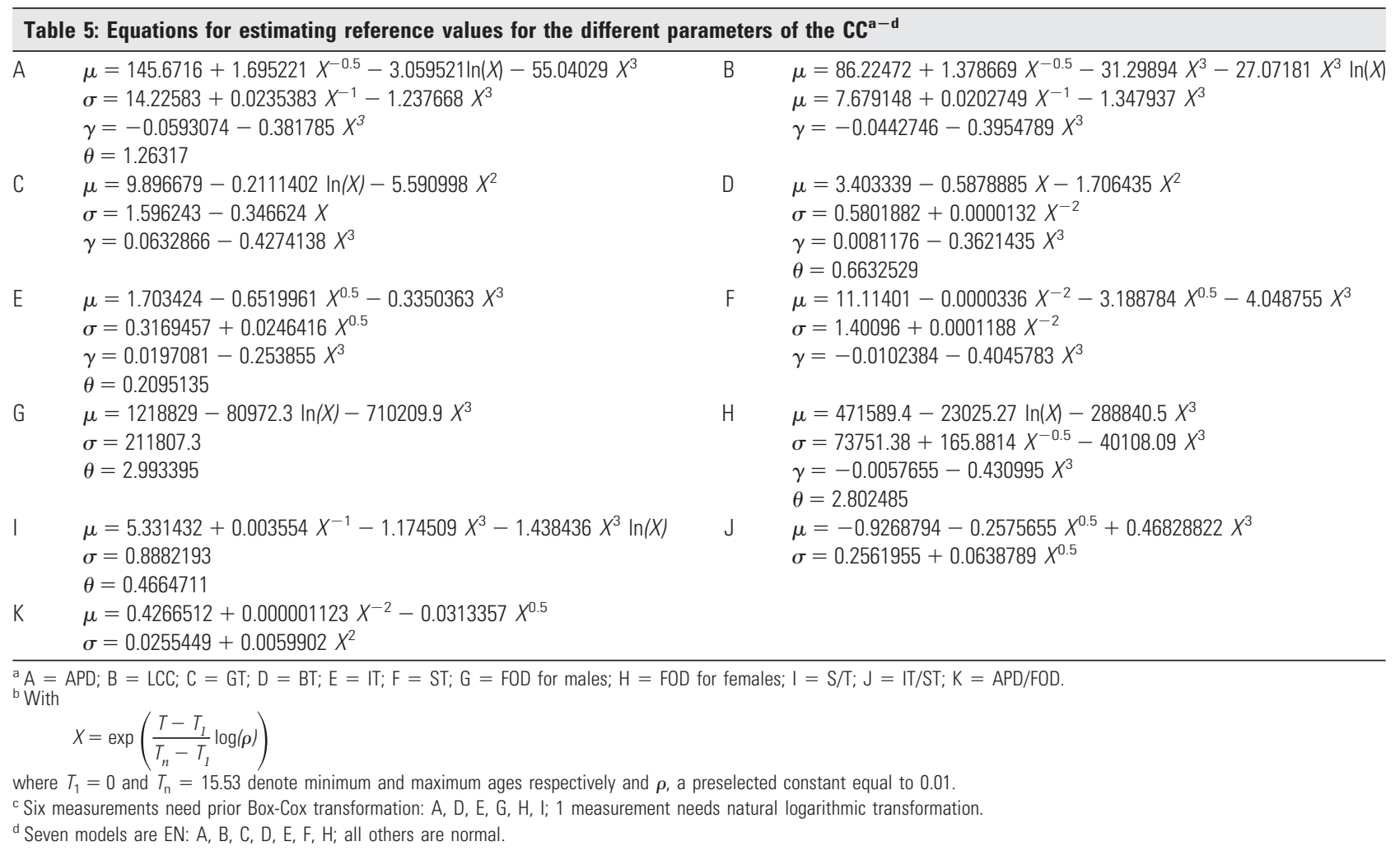

myelination of the axons crossing the CC. ${ }^{10}$ Subsequently, dysmyelinating and demyelinated disorders, pre- and perinatal trauma, or hypoxic injury is likely to be associated with changes in the thickness of the CC, involving either the whole CC in case of diffuse disorders or only part of it in case of localized damage. Thus, we excluded from this study children with such disorders.

The CC size is determined by the number and size of its constituent axons, myelination, and perivascular fluid. Cell death is responsible for the decrease in size of the CC. The eventual size and shape of each part of the CC are the result of a balance between cell death and increase in myelination and axon diameter. ${ }^{10-12}$ Many factors have been reported as implicated in CC growth and morphology.

The first factor is age: In fetuses, the anteroposterior diameter of the CC grows steadily. ${ }^{6,13}$ Modeling of the CC is not present, and the CC has a tubular appearance without isthmic narrowing. To our knowledge, very few studies have focused on CC growth in infants. It has been reported ${ }^{14}$ that a variable spurt of growth occurs in the genu during the second month and rapid growth occurs in the splenium between 4 and 6 months of age. Our results are not in keeping with these findings because GT and ST showed rapid growth until 3 years of age. Moreover, unlike what was observed by the same authors, ${ }^{14}$ we could not conclude that the CC showed an adult appearance by approximately 8 months of age. Our study was performed 20 years later, and the difference in image quality may account for this disagreement. In our study, stabilization of the IT/ST ratio after 3 years of age indicates that CC modeling is completed at that age and not earlier. The position of the $\mathrm{S} / \mathrm{T}$ also remained unchanged after 3 years of age. Our results are in keeping with studies evaluating CC growth in older children and confirm progressive increase in CC length throughout childhood. ${ }^{10,15}$ However, unlike these authors, we could not observe a predominant increase in CC posterior subregions (ST in our study) during childhood and adolescence.

The second factor is prematurity: In adolescents born very preterm (before 33 weeks' gestation), the total cross-sectional area of the CC has been reported to be significantly smaller than that in term children. ${ }^{11,16}$ Moreover, when considering subregions of the CC, the same authors ${ }^{16}$ showed differences in the size of CC subregions: The adolescents who were born very preterm showed a decrease in the posterior quarter and enlargement of the anterior quarter of the CC, in the absence of obvious cerebral damage related to prematurity. The later development of the splenium would account for a higher sensitivity to such damage even when it is not depicted by imaging. Therefore, premature children were excluded from our study.

The third factor is genetics: Influence of genetics on the CC size and morphology is consistent with reports showing greater similarity in CC morphology in monozygotic twins. ${ }^{17}$

The fourth factor is sex: There are disagreements in the literature regarding this point. The first report suggesting a possible sex effect on CC morphology was published in $1982^{18}$ and described the splenium as being more prominent in female adults. Since that report, many studies have focused on that particular point and could not find any sex differences in the splenium size in adults ${ }^{19,20}$ or children. ${ }^{10,21}$ Our results are in keeping with these findings. In fetuses, the midbody of the CC has been reported to be thicker in females. ${ }^{22}$ In our study, there was no difference regarding BT in boys and girls. We found a statistically higher FOD in boys from 1 year of age but not statistically higher APD and LCC. However, it has been reported that adult men had larger ${ }^{23}$ and longer ${ }^{20} \mathrm{CCs}$ than women. 
The fifth factor is handedness: Non-right-handers have been reported to have larger CCs, especially in the anterior half $^{24}$ and especially in males. Handedness was not recorded in our study.

The CC shows a very high individual variation in its morphology. It may have a bulbous appearance due a marked widening of the splenium or it may be more tubular, with absent or slight narrowing at the level of the isthmus. ${ }^{20}$ This high morphologic variability accounts for possible difficulties in determining CC size. In the literature, several authors have used different techniques to estimate CC size and growth. Measurements were always acquired on a midsagittal section. Certain authors measured the sagittal surface area of the CC and subdivided the CC into $3,{ }^{20,23} 4,{ }^{16} 6,{ }^{24}$ or $7^{10,15,21}$ subregions to evaluate the growth of the whole CC and of each subregion. Other authors have also measured the anteroposterior length of 4 subregions ${ }^{12}$ or the perimeter of the CC. ${ }^{10,15}$

Distances measured between different landmarks identified on the CC have also been evaluated. ${ }^{19}$ These techniques are useful for a better understanding of CC growth and variations, but their main drawback is that they are not at all easy to use in daily practice. Therefore, we favored linear easily reproducible measurements. Many studies suggest a relationship between performance in cognitive tasks and CC size and morphology. ${ }^{25}$ The CC is probably involved in the acquisition of cognitive skills in adolescents. ${ }^{26} \mathrm{~A}$ study conducted in adolescents born very preterm (before 33 weeks' gestation) has demonstrated involvement of the CC in speech and language function, with a positive correlation between verbal intelligence quotient scores and the size of the posterior half of the CC, supporting the hypothesis that the CC may be involved in high-order cognitive processes. ${ }^{16}$

Changes in morphology and/or size of the CC have also been reported in childhood neuropsychiatric disorders, such as attention deficit/hyperactivity disorder, ${ }^{27,28}$ autism, ${ }^{29}$ or dyslexia. ${ }^{30}$ Therefore, evaluating CC biometry and morphology is useful in children presenting with neuropsychiatric disorders, but for this reason, children with such disabilities were excluded from our study.

\section{Conclusions}

We have provided the first reference biometric data of the CC in MR imaging, from birth to 15 years of age drawn from a large cohort of children. These measurements are reproducible and easy to use in daily practice. Moreover, morphologic CC changes throughout infancy, childhood, and adolescence can be depicted by using these data. Most parameters showed rapid growth until 3 years of age and then slower growth. No sex effect could be observed in the size or morphology of the CC. In the future, the use of these data will allow evaluating a possible correlation between CC biometry and morphology and the clinical status of children with disorders possibly associated with CC abnormalities (neuropsychiatric, dysmyelinating, demyelinating, and hypoxic-ischemic disorders and perinatal trauma).

\section{References}

1. Royston P, Wright EM. A method for estimating age-specific reference intervals ('normal ranges') based on fractional polynomials and exponential transformation. J R Statist Soc A 1998;161:79-101
2. Royston P, Wright EM. Goodness-of-fit statistics for age-specific reference intervals. Stat Med 2000;19:2943-62

3. Borghi E, de Onis M, Garza C, et al. Construction of the World Health Organization child growth standards: selection of methods for attained growth curves. Stat Med 2006;25:247-65

4. Rakic P, Yakovlev PI. Development of the corpus callosum and cavum septi in man. J Comp Neurol 1968;132:45-72

5. Griffiths PD, Batty R, Reeves MJ, et al. Imaging the corpus callosum, septum pellucidum and fornix in children: normal anatomy and variations of normality. Neuroradiology 2009;51:337-45

6. Tilea B, Alberti C, Adamsbaum C, et al. Cerebral biometry in fetal magnetic resonance imaging: new reference data. Ultrasound Obstet Gynecol 2009;33:173-81

7. Gilmore JH, Lin W, Corouge I, et al. Early postnatal development of corpus callosum and corticospinal white matter assessed with quantitative tractography. AJNR Am J Neuroradiol 2007;28:1789-95

8. Hermoye L, Saint-Martin C, Cosnard G, et al. Pediatric diffusion tensor imaging: normal database and observation of the white matter maturation in early childhood. Neuroimage 2006;29:493-504

9. Raybaud C. The corpus callosum, the other great forebrain commissures, and the septum pellucidum: anatomy, development, and malformation. Neuroradiology 52:447-77

10. Giedd JN, Rumsey JM, Castellanos FX, et al. A quantitative MRI study of the corpus callosum in children and adolescents. Brain Res Dev Brain Res 1996;91:274-80

11. Allin M, Nosarti C, Narberhaus A, et al. Growth of the corpus callosum in adolescents born preterm. Arch Pediatr Adolesc Med 2007;161:1183-89

12. Keshavan MS, Diwadkar VA, DeBellis M, et al. Development of the corpus callosum in childhood, adolescence and early adulthood. Life Sci 2002;70:1909-22

13. Achiron R, Achiron A. Development of the human fetal corpus callosum: a high-resolution, cross-sectional sonographic study. Ultrasound Obstet Gynecol 2001;18:343-47

14. Barkovich AJ, Kjos BO. Normal postnatal development of the corpus callosum as demonstrated by MR imaging. AJNR Am J Neuroradiol 1988;9:487-91

15. Giedd JN, Blumenthal J, Jeffries NO, et al. Development of the human corpus callosum during childhood and adolescence: a longitudinal MRI study. Prog Neuropsychopharmacol Biol Psychiatry 1999;23:571-88

16. Nosarti C, Rushe TM, Woodruff PW, et al. Corpus callosum size and very preterm birth: relationship to neuropsychological outcome. Brain 2004;127:2080-89

17. Oppenheim JS, Skerry JE, Tramo MJ, et al. Magnetic resonance imaging morphology of the corpus callosum in monozygotic twins. Ann Neurol 1989;26:100-04

18. DeLacoste-Utamsing C, Holloway RL. Sexual dimorphism in the human corpus callosum. Science 1982;216:1431-32

19. Ozdemir ST, Ercan I, Sevinc O, et al. Statistical shape analysis of differences in the shape of the corpus callosum between genders. Anat Rec (Hoboken) 2007;290:825-30

20. Suganthy J, Raghuram L, Antonisamy B, et al. Gender- and age-related differences in the morphology of the corpus callosum. Clin Anat 2003;16:396-403

21. Rajapakse JC, Giedd JN, Rumsey JM, et al. Regional MRI measurements of the corpus callosum: a methodological and developmental study. Brain Dev 1996;18:379-88

22. Achiron R, Lipitz S, Achiron A. Sex-related differences in the development of the human fetal corpus callosum: in utero ultrasonographic study. Prenat Diagn 2001;21:116-20

23. Sullivan EV, Rosenbloom MJ, Desmond JE, et al. Sex differences in corpus callosum size: relationship to age and intracranial size. Neurobiol Aging 2001;22:603-11

24. Habib M, Gayraud D, Oliva A, et al. Effects of handedness and sex on the morphology of the corpus callosum: a study with brain magnetic resonance imaging. Brain Cogn 1991;16:41-61

25. Hutchinson AD, Mathias JL, Jacobson BL, et al. Relationship between intelligence and the size and composition of the corpus callosum. Exp Brain Res 2009;192:455-64

26. Pujol J, Vendrell P, Junque C, et al. When does human brain development end? Evidence of corpus callosum growth up to adulthood. Ann Neurol 1993;34:71-75

27. Giedd JN, Castellanos FX, Casey BJ, et al. Quantitative morphology of the corpus callosum in attention deficit hyperactivity disorder. Am J Psychiatry 1994;151:665-69

28. Hill DE, Yeo RA, Campbell RA, et al. Magnetic resonance imaging correlates of attention-deficit/hyperactivity disorder in children. Neuropsychology 2003;17:496-506

29. Hardan AY, Pabalan M, Gupta N, et al. Corpus callosum volume in children with autism. Psychiatry Res 2009;174:57-61

30. von Plessen K, Lundervold A, Duta N, et al. Less developed corpus callosum in dyslexic subjects: a structural MRI study. Neuropsychologia 2002;40:1035-44 